\title{
Before Meals
}

National Cancer Institute

\section{Source}

National Cancer Institute. Before Meals. NCI Thesaurus. Code C64592.

Prior to ingestion of food. 